\title{
Commentary: Preconditioning tDCS facilitates subsequent tDCS effect on skill acquisition in older adults
}

\author{
George M. Opie ${ }^{1 *}$ and John Cirillo ${ }^{2,3}$ \\ ${ }^{1}$ Discipline of Physiology, Adelaide Medical School, The University of Adelaide, Adelaide, SA, Australia, ${ }^{2}$ Movement \\ Neuroscience Laboratory, Department of Exercise Sciences, The University of Auckland, Auckland, New Zealand, ${ }^{3}$ Centre for \\ Brain Research, The University of Auckland, Auckland, New Zealand
}

Keywords: metaplasticity, aging, TMS/tDCS, motor training, neuroplasticity

\section{A commentary on}

Preconditioning tDCS facilitates subsequent tDCS effect on skill acquisition in older adults by Fujiyama, H., Hinder, M. R., Barzideh, A., Van De Vijver, C., Badache, A. C., Manrique-C, M. N., et al. (2017). Neurobiol. Aging 51, 31-42. doi: 10.1016/j.neurobiolaging.2016.11.012

Long-term potentiation and depression (LTP and LTD) represent powerful means of altering synaptic communication. However, they have an inherent potential to excessively modulate neuronal excitability, possibly resulting in unfavorable destabilization of neural function. It has been suggested that homeostatic regulation (i.e., metaplasticity) of neuronal networks avoids such destabilization. This construct proposes that the threshold for modification of neuronal excitability is based on previous post-synaptic activity, with an elevated threshold following high

OPEN ACCESS

Edited by:

P. Hemachandra Reddy,

Texas Tech University Health Sciences

Center, USA

Reviewed by:

Arubala Reddy,

Texas Tech University Health Sciences

Center, USA

Jasvinder Singh Bhatti,

SGGS College, India

*Correspondence:

George M. Opie

george.opie@adelaide.edu.au

Received: 11 January 2017 Accepted: 17 March 2017

Published: 31 March 2017

Citation:

Opie GM and Cirillo J (2017) Commentary: Preconditioning tDCS facilitates subsequent tDCS effect on

skill acquisition in older adults.

Front. Aging Neurosci. 9:84.

doi: 10.3389/fnagi.2017.00084 neuronal activity, but a reduced threshold following low neuronal activity (Bienenstock et al., 1982). Studies using non-invasive brain stimulation (NIBS) over human primary motor cortex (M1) support the utilization of such a mechanism (Karabanov et al., 2015). Furthermore, NIBS plasticity interventions have demonstrated an ability to interact homeostatically with motor learning, a process that is at least partially LTP-dependent (Ziemann et al., 2004; Jung and Ziemann, 2009). This has led to the exciting possibility of using "priming" NIBS to enhance the response to motor training following injury, or compensate for deficits in neuroplastic capacity. While relevant to many different populations, such an approach is of particular interest in older adults, where deficits in neuroplastic capacity (Barnes, 2003) and motor learning (Voelcker-Rehage et al., 2006) are well defined. However, it is currently unknown whether priming stimulation remains effective for potentiating the response to training in the elderly.

In a recent paper within Neurobiology of Aging, Fujiyama and colleagues (Fujiyama et al., 2017) attempted to address this gap in knowledge by utilizing transcranial direct current stimulation (tDCS; a NIBS technique able to reliably induce LTP- and LTD-like plasticity in M1) to prime skill acquisition in young and older adults. The study utilized a visuomotor training task that involved moving a cursor on a computer screen through a range of targets by abducting the nondominant index finger against a force transducer. Each target required a different level of force and target order was consistent across trials. During training, all subjects received 20 mins of excitatory anodal tDCS (atDCS). However, prior to training, half received $10 \mathrm{~min}$ of inhibitory cathodal tDCS (ctDCS), whereas the other half received 10 min of sham stimulation. Single- and paired-pulse TMS over the right M1 was used to quantify corticomotor excitability (motor evoked potential (MEP) area), and intracortical function (short-interval intracortical inhibition, SICI; intracortical facilitation, ICF), respectively. Changes in neurophysiological measures and motor performance were assessed as outcome variables. The results indicated that both young and older 
adults improved in performance following training, with a greater magnitude of effect observed in young subjects. Furthermore, performance following priming ctDCS was greater than sham in both groups. In addition, motor training potentiated corticospinal excitability in both groups, but only when preceded by ctDCS. In contrast, there was no modulation of ICF or SICI following motor training. The authors suggest that these findings indicate a maintenance of metaplastic mechanisms in the elderly, while also demonstrating the utility of priming stimulation for improving the response to training in older adults.

While Fujiyama and colleagues are the first to investigate whether aging impairs the capacity of priming stimulation to modulate the response to motor training, another recent study used paired blocks of theta burst stimulation (TBS; a TMS paradigm able to induce LTP- and LTD-like changes in corticospinal excitability) to investigate age-related changes in the mechanisms of metaplasticity (Opie et al., 2017). In contrast to Fujiyama et al., this study reported that priming was ineffective in older adults. The cause of these discrepant findings is currently unclear. However, differences in the effectiveness of the applied NIBS techniques for modulating excitability in M1 may be a factor, possibly suggesting a greater clinical utility of tDCS for the metaplastic modulation of skill acquisition in older adults. One factor contributing to this outcome may be that tDCS was applied during task performance, whereas TBS was applied at rest. Subsequently, tDCS was given at a time when neural activation of task-related muscles was increased to successfully execute the movement, which likely influenced the plasticity response (Ridding and Ziemann, 2010). In addition, the conventional tDCS montage used by Fujiyama et al. is less focal (i.e., stimulates a larger cortical area) than other NIBS techniques (such as TBS), and may have resulted in activation of cortical networks other than M1 that could be important in motor learning. Therefore, the metaplastic modulation of corticospinal excitability observed by Fujiyama and colleagues may have stemmed from their utilized intervention producing a stronger and more widespread activation of motor cortical networks. The conflicting findings between these studies may therefore indicate that the aging

\section{REFERENCES}

Barnes, C. A. (2003). Long-term potentiation and the ageing brain. Philos. Trans. R. Soc. Lond. B Biol. Sci. 358, 765-772. doi: 10.1098/rstb.2002.1244

Bienenstock, E. L., Cooper, L. N., and Munro, P. W. (1982). Theory for the development of neuron selectivity: orientation specificity and binocular interaction in visual cortex. J. Neurosci. 2, 32-48.

Fujiyama, H., Hinder, M. R., Barzideh, A., Van De Vijver, C., Badache, A. C., Manrique-C, M. N., et al. (2017). Preconditioning tDCS facilitates subsequent tDCS effect on skill acquisition in older adults. Neurobiol. Aging 51, 31-42. doi: 10.1016/j.neurobiolaging.2016.11.012

Jung, P., and Ziemann, U. (2009). Homeostatic and nonhomeostatic modulation of learning in human motor cortex. J. Neurosci. 29, 5597-5604. doi: 10.1523/JNEUROSCI.0222-09.2009

Karabanov, A., Ziemann, U., Hamada, M., George, M. S., Quartarone, A., Classen, J., et al. (2015). Consensus paper: probing homeostatic plasticity of human cortex with non-invasive transcranial brain stimulation. Brain Stimul. 8, 993-1006. doi: 10.1016/j.brs.2015.06.017 process results in an increased threshold for inducing metaplastic effects.

While the cause of these conflicting findings may be interesting, the real challenge of priming interventions is ensuring that whether motor improvements observed within the lab can be functionally translated to everyday life. This question highlights the volume of work that remains to fully elucidate the role that priming stimulation may have in the field of aging. There are several parameters that require investigation to help answer this question. For example, can motor improvement in older adults be made stronger, more stable or longer lasting by applying additional blocks of priming stimulation? How critical is the lapsed time between priming and training? Are alternative approaches that change neuronal excitability immediately prior to modulating synaptic strength (a notion referred to as "gating" that reflects a non-homeostatic effect of excitabilityenhancement) (Ziemann and Siebner, 2008) more effective for potentiating the plasticity response? Can homeostatic and non-homeostatic mechanisms be combined with metaplastic principles to produce an optimal outcome? Despite the current uncertainty surrounding priming interventions and their functional relevance with advancing age, the findings of Fujiyama and colleagues demonstrate that it is possible to metaplastically potentiate the response to motor training in older adults. While the findings should be considered preliminary until repeated within a larger cohort using a between-subjects design, they represent an interesting and exciting contribution to the field. Ultimately, identifying metaplastic processes optimal for motor learning in older adults may offer novel insights about the use of priming approaches for neurorehabilitation.

\section{AUTHOR CONTRIBUTIONS}

GO and JC both conceptualized, drafted, revised, and approved manuscript.

\section{ACKNOWLEDGMENTS}

The authors kindly acknowledge Associate Professor John Semmler for his feedback and advice.

Opie, G. M., Vosnakis, E., Ridding, M. C., Ziemann, U., and Semmler, J. G. (2017). Priming theta burst stimulation enhances motor cortex plasticity in young but not old adults. Brain Stimul. 10, 298-304. doi: 10.1016/j.brs.2017. 01.003

Ridding, M. C., and Ziemann, U. (2010). Determinants of the induction of cortical plasticity by non-invasive brain stimulation in healthy subjects. J. Physiol. 588, 2291-2304. doi: 10.1113/jphysiol.2010.190314

Voelcker-Rehage, C., Stronge, A. J., and Alberts, J. L. (2006). Age-related differences in working memory and force control under dual-task conditions. Aging Neuropsychol. Cogn. 13, 366-384. doi: 10.1080/1382558909 69339

Ziemann, U., Ilic, T. V., Pauli, C., Meintzschel, F., and Ruge, D. (2004). Learning modifies subsequent induction of long-term potentiation-like and long-term depression-like plasticity in human motor cortex. J. Neurosci. 24, 1666-1672. doi: 10.1523/JNEUROSCI.5016-03.2004

Ziemann, U., and Siebner, H. R. (2008). Modifying motor learning through gating and homeostatic metaplasticity. Brain Stimul. 1, 60-66. doi: 10.1016/j.brs.2007.08.003 
Conflict of Interest Statement: The authors declare that the research was conducted in the absence of any commercial or financial relationships that could be construed as a potential conflict of interest.

The reviewer AR and handling Editor declared their shared affiliation, and the handling Editor states that the process nevertheless met the standards of a fair and objective review.
Copyright (c) 2017 Opie and Cirillo. This is an open-access article distributed under the terms of the Creative Commons Attribution License (CC BY). The use, distribution or reproduction in other forums is permitted, provided the original author(s) or licensor are credited and that the original publication in this journal is cited, in accordance with accepted academic practice. No use, distribution or reproduction is permitted which does not comply with these terms. 